\title{
Évaluer pour piloter
}

Réflexion sur le cas français

Evaluation as an adjunct to education planning. The French case

Evaluar para guiar. La reflexión en el caso francés

\section{Alain Michel}

\section{OpenEdition \\ Journals}

Édition électronique

URL : http://journals.openedition.org/ries/2521

DOI : $10.4000 /$ ries. 2521

ISSN : 2261-4265

Éditeur

Centre international d'études pédagogiques

Édition imprimée

Date de publication : 1 juin 2000

Pagination : 19-29

ISSN : 1254-4590

Référence électronique

Alain Michel, «Évaluer pour piloter », Revue internationale d'éducation de Sèvres [En ligne], 26 l juin 2000, mis en ligne le 01 juin 2003, consulté le 02 mai 2019. URL : http://journals.openedition.org/ries/2521 ; DOI : 10.4000/ries.2521 


\title{
Évaluer pour piloter
}

\section{Réflexion sur le cas français}

\author{
Alain Michel
}

\section{Résumé}

Le caractère polysémique du terme "évaluation» incite à nuancer son usage. Les conceptions de l'évaluation varient d'ailleurs d'un pays à l'autre. Au sein d'un même système éducatif, les niveaux d'interprétation different sensiblement. On comprend mieux les réticences, voire l'hostilité de nombreux partenaires du système éducatif face aux diverses modalités de l'évaluation. C'est pourtant, grâce à une circulation accélérée et permanente de l'information, la seule voie actuellement offerte à une évolution réelle et continue de l'éducation.

\section{Evaluation as an adjunct to education planning. The French case}

The polysemous nature of the term "evaluation» requires some care in its use. Evaluation notions vary from one country to the next. Within the same education system, the extent to which this term is interpreted may vary greatly. This in turn helps to explain the reluctance, or even hostility, of certain actors in education vis-à-vis the relative diversity of evaluation methods in use. Nonetheless, the provision of easily accessible and up-to-date information is the only way to achieve continuous and lasting improvements in education.

\section{Evaluar para guiar. La reflexión en el caso francés}

El carácter polisémico del término kevaluación» instiga a matizar su empleo. Además, cada país tiene su propia concepción de la evaluación y, dentro de un sistema educativo, los niveles de interpretación ya difieren notablemente. Entonces, cualquiera comprende mejor las reticencias, o la hostilidad que sienten varios interventores del sistema educativo acerca de las diversas modalidades de la evaluación. Una transmisión acelerada y permanente de la información es actualmente el único camino que puede tomar una evolución real y continua de la educación. 
L'évaluation a le vent en poupe, depuis plus d'une décennie, au sein des systèmes éducatifs des pays industrialisés. Cette émergence d'une nouvelle «culture de l'évaluation»' s'est inscrite dans un mouvement plus large concernant l'ensemble des services publics. C'est ce que les Anglo-Saxons ont appelé the demand for accountability et les Français, l'attente de transparence par les usagers à l'égard du fonctionnement du service public et de ses résultats. Il s'agit là d'un premier facteur déterminant à l'origine de ce mouvement. Le souci de maîtrise des dépenses publiques a joué un rôle de premier plan. Ce souci appelle à davantage d'efficience et d'efficacité, donc à de nouveaux instruments de mesure des résultats, y compris dans des domaines traditionnellement réticents aux approches quantitatives, comme la santé, l'environnement ou l'éducation. Un autre facteur est lié au processus de décentralisation des décisions et à l'objectif de donner davantage d'autonomie aux acteurs du terrain, au sein d'un monde en mutation rapide. C'est la remise en cause des modèles tayloriens et bureaucratiques d'organisation

L'évaluation ne doit être qu'un instrument au service de davantage de démocratie et de concertation, un outil d'aide à la négociation et à la décision

et l'avènement d'un management participatif à travers lequel il s'agit de favoriser les initiatives du terrain et les innovations. Mais les décisions des acteurs doivent être cohérentes et en matière de service public, où ne joue pas la sanction du marché, il convient
contrôle du bien-fondé des décisions d'inventer de nouveaux instruments de contrôle du bien-fondé des décisions prises et des modes de fonctionnement des unités devenues plus autonomes. Faute de «main invisible» régulatrice, il faut créer de nouveaux dispositifs de régulation et de pilotage. À une régulation de type bureaucratique et de contrôle a priori, notamment de normes de fonctionnement, se substitue une régulation de type cybernétique, fondée sur la circulation rapide de l'information pertinente, y compris sur les résultats obtenus, dans le cadre de procédures contractuelles. C'est dans ce contexte qu'est apparue, au début des années quatre-vingt-dix, l'expression de "pilotage» de l'établissement scolaire ou du système éducatif ${ }^{2}$.

\section{L'émergence d'une nouvelle culture d'évaluation}

Dans un contexte mondial de décalage croissant entre l'offre et la demande sociale d'éducation, il a donc fallu faire face à une triple exigence de transparence démocratique, d'efficacité et d'équité. À la réalité objective de la

\footnotetext{
1 A. Michel, "Vers une nouvelle culture de l'évaluation », Éducation et management, novembre 1992 ; C. Thélot, L'évaluation du système éducatif, Nathan, décembre 1993 ; P. Broadfoot, "Un nouveau mode de régulation dans un système décentralisé : l'État évaluateur », Revue française de pédagogie, $n^{\circ} 130,2000$.

2 A. Michel, "Pilotage d'un système complexe : l'Éducation nationale ", Administration et éducation, n², 1993 ou " La conducción de un sistema complejo: la educación nacional », Revista iberoamericana de educación, avril 1996.
} 
"crise mondiale de l'éducation» diagnostiquée dès les années soixante ${ }^{3}$, s'est superposée une crise de confiance, y compris dans les pays les plus développés. À cet égard, la publication en 1983, aux États-Unis, du rapport A Nation at Risk (Une nation en danger) qui dénonçait l'inefficacité de l'école américaine, a eu une répercussion mondiale et a conduit le gouvernement américain à financer de grandes enquêtes internationales sur les acquis des élèves et à élaborer des indicateurs. Ces initiatives ont été relayées par les organisations internationales (OCDE et UNESCO) et par les divers pays développés. La publication par l'UNESCO, en 1991, du premier Rapport mondial sur l'éducation et celle en 1993, par l'OCDE, de la première version de Regards sur l'éducation (Education at a Glance), premier ensemble d'indicateurs internationaux sur l'éducation, ont constitué des étapes décisives dans la voie d'une évaluation comparative. Bien entendu, il faut aussi mentionner les grandes enquêtes internationales sur les résultats des élèves, conduites par les deux grands opérateurs internationaux : l'ETS ${ }^{4}$ et l'IEA ${ }^{5}$.

Mais l'évaluation dans le champ de l'éducation pose des problèmes particuliers qu'il convient de rappeler brièvement. En premier lieu, l'évaluation a toujours été au centre des pratiques éducatives. Si bien que beaucoup d'enseignants se demandent ce qui est vraiment nouveau dans le discours sur l'évaluation, sinon le fait de conceptualiser certaines distinctions - comme l'évaluation diagnostique, formative ou formatrice, sommative ou certificative - et d'avoir davantage recours à des tests standardisés, dont certains soupçonnent qu'ils sont à la maïeutique socratique ce que le hamburger est à la blanquette de veau des restaurants traditionnels. C'est l'effet Jourdain : "L'évaluation, on la pratique depuis toujours, sans vraiment en percevoir toute la saveur et toutes les implications.» Ce sentiment nourrit souvent une méfiance, voire une hostilité, à l'égard de l'élaboration de tests standardisés. Il est vrai que certains excès des approches des psychométriciens et un risque réel de dérive nominaliste semblent justifier une résistance des enseignants. On ne peut, en effet, rendre compte en termes quantitatifs de l'ensemble des acquis cognitifs et non cognitifs des élèves. Mais, dans le même temps, les diverses études de docimologie ont montré, depuis longtemps, la très grande subjectivité des critères d'appréciation des professeurs et les risques importants qui en découlent, tant du point de vue de l'efficacité que de l'équité ${ }^{6}$. Il reste que le nécessaire dessein de disposer de points de repère plus objectifs doit cependant prendre en compte l'aversion des enseignants pour des outils d'évaluation normalisés.

3 P.H. Coombs, La crise mondiale de l'éducation, Bruxelles, De Boeck Université, 1989.

4 Educational Testing Service.

5 International Association for the Evaluation of Educational Achievement.

6 P. Merle, Sociologie de l'évaluation scolaire, Puf, 1998 ; OCDE, Performance Standards in Education, 1995 ; A.

Duru-Bellat, A. Van Zanten, Sociologie de l'école, Armand Colin, 1999. 
Par ailleurs, l'évaluation est polysémique et entraîne de nombreuses confusions dans les esprits. La démarche évaluative peut répondre à des finalités diverses et concerner des objets différents. En particulier, il faut distinguer l'évaluation des acquis des élèves, de celle des personnels et des établissements scolaires, ou encore l'évaluation de l'état d'un système à un moment donné de celle d'une innovation ou d'une politique. Chacun perçoit l'évaluation en fonction de son rôle (en tant qu'acteur, usager ou partenaire) et de ses attentes. Toute démarche évaluative doit donc être accompagnée, ou mieux précédée, d'une explicitation de ses objectifs et de ses fondements, mais aussi associer le plus en amont possible l'ensemble des acteurs et des partenaires concernés. La clarification des règles du jeu est une condition essentielle du bon déroulement des procédures d'évaluation et de la pertinence de l'évaluation comme instrument de pilotage à divers niveaux : depuis la classe jusqu'au niveau national ou supranational, en passant par les niveaux intermédiaires (académies, régions, établissements scolaires, etc.). Notre propos est ici de se centrer sur le rôle fondamental que peut et doit jouer l'évaluation, sous ses divers aspects, comme instrument de pilotage dans le contexte d'une stratégie systémique de changement.

\section{Piloter le changement}

Dans un monde en mutation rapide, l'école est sommée d'évoluer, à la fois pour s'adapter aux modifications de son environnement et pour préparer les jeunes et les moins jeunes à un avenir de moins en moins prévisible. L'école doit à la fois modifier les programmes (le curriculum) et ses modes de fonctionnement. En particulier, la règle des trois unités (temps, lieu, action) de la tragédie classique, qui se sont longtemps appliquées dans l'univers scolaire, sont devenues caduques en raison des nouveaux défis à relever et des nouvelles attentes à l'égard de l'éducation? . Désormais, on apprendra à tout âge, de plus en plus en dehors de l'école, avec divers partenaires et pas seulement des enseignants. Dans un tel contexte, l'école devra redéfinir sa spécificité et devenir elle-même une organisation apprenante.

Dans ce mouvement perpétuel, il est apparu, dans tous les pays, que la succession des réformes décidées du sommet, au rythme des changements de ministre, présentait plus d'inconvénients que d'avantages. On ne change pas l'école par décret, surtout sans un soutien des principaux acteurs, c'est-à-dire les enseignants. Mais, à l'opposé, le fait de donner davantage d'autonomie aux établissements scolaires ne garantit pas une évolution conforme à quelques objectifs essentiels définis au niveau politique le plus élevé. D’une part, certaines innovations nées dans un contexte particulier ont du mal à se généraliser dans des contextes différents, d'autre part la diversité souhaitable qui peut résulter des

7 A. Michel, "Une école pour un monde nouveau ", Futuribles, $n^{\circ} 252$, avril 2000. 
initiatives locales ne doit pas conduire à des inégalités inacceptables ${ }^{8}$. De fait, les grandes enquêtes internationales sur la capacité de lecture ou sur les acquis des élèves en mathématiques et en sciences ont montré qu'il n'y avait pas de corrélation entre le niveau plus ou moins élevé de décentralisation des systèmes éducatifs et le niveau des élèves, mais que la variance des résultats (entre élèves et entre établissements) était plus grande dans les systèmes les plus décentralisés ${ }^{9}$. Dès lors que l'on considère que l'école doit plutôt réduire les inégalités que les reproduire ou les accentuer, il convient donc de fixer un cadre national ferme, comme le préconisent $\mathrm{P}$. Joutard et $\mathrm{C}$. Thélot, pour réguler le fonctionnement du système éducatif, afin de concilier une plus grande autonomie des acteurs, donc une diversification souhaitable des parcours scolaires et des approches pédagogiques, et l'objectif d'égalité des chances.

Du reste, on constate au niveau international un double mouvement : les pays les plus décentralisés tendent à se recentraliser (curriculum national, tests nationaux standardisés), c'est le cas, par exemple, des États-Unis et du Royaume-Uni, tandis que des pays encore relativement centralisés tendent à se décentraliser davantage ; c'est le cas, par exemple, de la France et du Portugal ${ }^{10}$. Chacun semble donc en quête d'un niveau optimal de décentralisation et du mode de régulation approprié à ses finalités et à sa culture nationale.

Dans une perspective dynamique, la question de la régulation du système éducatif devient celle du pilotage du changement, c'est-à-dire la recherche simultanée d'une cohérence synchronique et diachronique, ou en d'autres termes, la recherche d'un mode organisationnel permettant à la fois de faciliter les innovations et l'adaptation au local et d'assurer une "cohérence finalisée» à l'ensemble des microdécisions. Étant donné que la complexité d'un système augmente avec l'accroissement de la marge de liberté de ses acteurs, on se situe bien dans la problématique du pilotage d'un système complexe. Ainsi que l'a énoncé I. Prigogine, le maximum de complexité qu'un système peut atteindre sans devenir trop instable est directement fonction de la vitesse de circulation de l'information (nous ajoutons "pertinente») entre les diverses unités relativement autonomes composant le système. Ce qui suppose donc un bon système d'information statistique, des indicateurs d'évaluation, une fonction de prévision et de prospective, enfin une fonction de communication stratégique ${ }^{11}$. C'est dire que l'évaluation est un instrument essentiel de pilotage, mais pas le seul.

8 P. Joutard, C. Thélot, Réussir l'école. Pour une politique éducative, Paris, Seuil, 1999.

9 P. Black, A. Michel, Learning from Pupil Assessment: International Comparison, Los Angeles, Éd. U.C.L.A, 1998. 10 Voir articles de J. Barroso, de B. Davies et L. Ellison, et de A. Michel in The School between the Local and the Global. Perspectives on the 21st Century, Université de Lisbonne, octobre 1999 (ouvrage également publié en portugais) ; voir aussi J. Barroso, "Autonomie et modes de régulation locale dans le système éducatif ", Revue française de pédagogie, $\mathrm{n}^{\circ} 130,2000$.

$11 \mathrm{lbid}$. note 2 pour un développement de ces points. 
Autre point essentiel, le système éducatif est un système vivant ouvert sur son environnement, avec lequel il est en interaction dynamique permanente. C'est un «système auto-éco-organisateur» au sens d'Edgar Morin, c'est-à-dire un ensemble d'unités et d'acteurs liés par des échanges et capables en interaction avec leur environnement de réagir, d'évoluer, d'apprendre, d'inventer et de s'auto-organiser ${ }^{12}$. Cette métaphore du système vivant n'est pas un simple exercice intellectuel. Elle est directement utile pour penser la politique éducative, jusqu'ici implicitement fondée sur une vision mécaniste du système éducatif. En effet, le terme même de réforme, au sens où il est utilisé dans l'éducation, relève surtout de la logique de l'ingénieur. La réforme suppose que l'on connaisse bien le fonctionnement du système et que l'on soit capable de prévoir les résultats de sa transformation à une échéance plus ou moins précise. Il $y$ a là une certaine contradiction avec le souci de donner davantage d'autonomie aux acteurs et de développer diverses formes de contractualisation. Or, le changement est un processus complexe qui a tous les caractères d'une «aventure humaine», ce qui implique une bonne dose d'incertitude quant à son déroulement, même si un cap général est fixé en termes de grands objectifs à atteindre.

C'est pourquoi nous proposons d'abandonner la notion de «réforme» et de considérer le changement dans l'éducation comme un processus systémique et soutenable ${ }^{13}$. Le changement doit être conçu comme un processus, ce qui signifie que la dimension temps est essentielle et ne doit pas être pensée de manière uniforme sur l'ensemble du territoire : il doit y avoir diversité des rythmes en fonction des contextes. Ce processus doit être systémique pour deux raisons :

- la nécessité d'une cohérence finalisée des initiatives des acteurs ;

- les interactions entre les diverses dimensions de l'enseignement et de l'apprentissage : contenus des programmes, approches pédagogiques, organisation du temps et de l'espace scolaire, procédures et critères d'évaluation des élèves et des enseignants, utilisation des nouvelles technologies de l'information et de la communication (NTIC), formation initiale et continue des enseignants, modes de management des écoles et établissements scolaires, instruments de pilotage à tous les niveaux, etc.

Enfin, le processus doit être soutenable, car il doit être conçu comme une dynamique continue de rénovation à un rythme supportable par les acteurs du système, ce qui n'exclut pas des changements de rythme et des périodes plus calmes...

12 E. Morin, Introduction à la pensée complexe, Paris, ESF, 1990.

13 A. Michel, "Pour une approche systémique du changement ", Administration et éducation, n¹, 1996. 


\section{L'évaluation dans une approche systémique du changement}

L'évaluation concerne les divers niveaux de pilotage du système éducatif et peut obéir à des logiques différentes. L'évaluation des acquis cognitifs et non cognitifs des élèves est la plus couramment développée dans tous les pays, surtout dans les pays industrialisés, car elle apparaît comme étant susceptible de donner une indication sur l'efficacité des processus d'apprentissage. Sa finalité peut être à dominante formative ou sommative. Dans la classe, elle devrait être à dominante formative, car il s'agit pour le professeur et pour l'élève de disposer de points de repère relativement objectifs afin de déterminer les principales carences de l'élève, mais aussi de mesurer les progrès accomplis. Dans le contexte de l'enseignement de masse et du souci de lutter contre l'échec scolaire, car aujourd'hui le minimum de connaissances et de compétences requis pour s'insérer normalement dans la société et trouver un emploi est plus élevé que par le passé, l'importance de l'évaluation formative ne cesse de s'accroître. L'enseignant doit pouvoir gérer au mieux une hétérogénéité accrue des élèves (résultant elle-même de la fracture sociale) et amener chaque élève à un niveau minimum reconnu de qualification (objectif en France de la loi du 10 juillet 1989), car le diplôme est de plus en plus en nécessaire, même s'il est de moins en moins suffisant, pour trouver un emploi.

Mais les tests normalisés à finalité diagnostique ou formative, comme ceux qui ont été introduits en France à la fin des années quatre-vingt à l'entrée en CE2, au collège et au lycée, peuvent aussi être utilisés au niveau des écoles et des établissements, des académies et au niveau national, pour faire des comparaisons dans le temps et dans l'espace. L'agrégation à ces divers niveaux des résultats aux tests permet de fixer des priorités d'action et de politique. Encore faut-il que le constat des résultats ne soit pas considéré comme une fin en soi et que soient analysées les causes des résultats constatés. L'analyse de ces causes doit être une priorité de la recherche en éducation. Parmi les facteurs expliquant les résultats des élèves aux tests normalisés ou aux examens, certains sont directement liés aux comportements des élèves ou à la pédagogie du maître, mais d'autres sont liés à la vie scolaire ou à la politique de l'établissement (effet établissement) ; d'autres encore sont liés au contexte familial, social ou économique. C'est dire que l'évaluation ne saurait se limiter à élaborer des outils de mesure des acquis des élèves. Encore faut-il souligner l'intérêt à disposer de données comparatives au niveau international à travers les grandes enquêtes du type de celles de l'ETS et l'IEA. C'est précisément l'objectif du Centre pour la recherche et l'innovation dans l'enseignement (CERI) de l'OCDE qui a établi un dispositif international capable de fournir régulièrement des données de ce type par l'organisation de grandes enquêtes internationales à intervalles réguliers. De telles enquêtes (ce fut 
le cas de TIMSS : troisième enquête internationale sur les acquis en mathématiques et en sciences) comportent, outre des banques d'items, des questionnaires très élaborés à l'intention des élèves et des enseignants, afin de pouvoir analyser les facteurs expliquant la diversité des résultats obtenus.

La pluralité des facteurs pouvant intervenir nécessite des analyses statistiques sophistiquées (analyse factorielle, analyse multivariée, etc.) mais aussi des études qualitatives en profondeur (recherche de «métadonnées») qui permettent de mieux comprendre les interactions complexes entrant en jeu. D'où la nécessaire complémentarité de l'évaluation quantitative à partir de statistiques ou d'indicateurs et de l'évaluation qualitative qui peut être accomplie sous forme d'inspections ou d'audits. Cette complémentarité s'impose particulièrement au niveau de l'établissement scolaire ou de l'académie. Étant donné la mise en évidence par les sciences de l'éducation de l'importance de "l'effet établissement ", un effort particulier a été accompli durant ces dernières années pour développer des indicateurs à ce niveau et pour élaborer des méthodes d'audit adaptées à l'univers scolaire. Ainsi, en France, les indicateurs de pilotage des établissements scolaires (IPES), mais aussi des opérations d'audit, y compris à l'échelle d'une académie entière (l'académie de Lille), ont-ils été développés par le ministère de l'Éducation nationale. Les IPES constituent des outils permettant d'éclairer les discussions et les choix à la base d'un projet d'établissement ${ }^{14}$. S'ils sont de plus en plus utilisés par les chefs d'établissement ou leurs adjoints, il ne faut pas nier la réticence de nombre d'entre eux à l'égard de tels instruments. Certes, ces outils ne sont pas la panacée et sont encore imparfaits ${ }^{15}$, mais ils permettent de disposer de données objectives utiles dans un milieu où règnent souvent des préjugés et des pétitions de principes. Ces IPES constituent donc, parmi d'autres documents, une base d'information pour une autoévaluation ainsi que pour un audit externe et permettent de gagner du temps dans l'organisation des entretiens lors d'un tel audit, en focalisant l'attention sur des points vulnérables ou névralgiques du fonctionnement de l'établissement observé. Il importe ici de souligner une autre complémentarité nécessaire : l'audit externe et l'autoévaluation. Diverses analyses des grandes opérations d'audit conduites ces dernières années en France sont disponibles ${ }^{16}$. Le mouvement est lancé et semble devoir se développer, notamment dans la perspective de l'audit conçu comme un levier du changement et une aide à la révision du projet d'établissement. Par ailleurs, cette évolution conduit à privilégier une approche globale de l'établissement, articu-

14 J-C. Emin, C. Sauvageot, "IPES, un dispositif d'indicateurs pour le pilotage des établissements scolaires ", Administration et éducation, $n^{\circ} 4,1995$; Les IPES, Ministère de l'Éducation nationale, mars 1998.

15 P. Merle, "Les IPES et l'analyse de l'efficacité : une articulation incertaine ", Administration et éducation, $n^{\circ} 4$, 1999.

16 F. Dubet, E . Bautier, M. Duru-Bellat, J-P. Payet, Synthèse de l'audit sur les collèges, MEN, mai 1999 ; L. Demailly et al., Évaluer les établissements scolaires : enjeux, expériences, débats, Paris, L'Harmattan, 1998. 
lant mieux que par le passé organisation et gestion de l'établissement, vie scolaire, enseignements disciplinaires et fonction documentaire. Elle conduit aussi à la poursuite de la redéfinition des missions des corps d'inspection : inspecteurs pédagogiques régionaux et inspecteurs généraux, appelés à travailler de plus en plus au sein d'équipes pluridisciplinaires et avec des inspecteurs généraux de l'administration. À cet égard, on constate une influence anglo-saxonne, notamment celle de l'OFSTED ${ }^{17}$ en Grande-Bretagne.

En France, la démarche d'audit a même été expérimentée au niveau académique et désormais chaque année quelques académies (de l'ordre de cinq) devraient être concernées. L'équipe d'audit comprend là aussi des inspecteurs pédagogiques et des inspecteurs de l'administration. On peut s'attendre à ce que de tels audits suscitent l'élaboration de nouveaux indicateurs au niveau des académies. Ces audits ne sont pas toujours bien perçus, car le diagnostic sur l'état d'une entité, à un moment donné, est souvent considéré, à tort, comme un jugement sur la qualité des responsables. Or, comme l'a bien souligné Claude Thélot ${ }^{18}$, il ne faut pas confondre l'évaluation de l'état d'un système à un moment donné avec l'évaluation d'une politique. De même, l'évaluation d'un établissement ne doit pas être confondue avec l'évaluation d'un proviseur ou d'un principal. Tant de facteurs entrent en jeu ! Certes, un audit et des indicateurs peuvent mettre en évidence des carences de management ou des erreurs politiques, mais il ne faut pas pour autant confondre les objectifs et les approches.

Un pas important a été franchi avec l'élaboration d'indicateurs au niveau national et international. En France, la publication annuelle depuis le début des années quatre-vingt-dix, par la direction de l'évaluation et la prospective (DEP) puis la direction de la programmation et du développement (DPD), de L'état de l'école - ensemble de trente indicateurs sur les ressources, les coûts, l'activité et les résultats du système éducatif français - est un élément important de transparence qui permet d'éclairer les grands débats sur l'éducation. La publication conjointe d'indicateurs au niveau des académies, Géographie de l'école, est un complément utile qui a permis aux académies de mieux situer leurs caractéristiques et leurs performances, les unes par rapport aux autres, et au gouvernement de mieux percevoir les enjeux éducatifs d'une politique d'aménagement du territoire. Ces informations, parmi d'autres, ont également permis de fonder de nouvelles relations contractuelles entre les académies et l'administration centrale du ministère.

Sur le plan international, le projet INES de l'OCDE, qui remonte à 1987 (première réunion de lancement à Poitiers), a permis de publier au début des années quatre-vingt-dix le premier ensemble international d'indicateurs sur 
l'éducation. La publication annuelle Regards sur l'éducation, accompagnée depuis quelques années d'une analyse de certains aspects des politiques éducatives, est le plus grand succès éditorial de l'OCDE. Dans le domaine de l'éducation, cette activité de l'OCDE est considérée comme prioritaire par l'ensemble des pays membres, soucieux de disposer d'éléments de comparaison sur les coûts, les taux de scolarisation et de diplômés à divers niveaux et sur les acquis des élèves. Le projet INES a permis de développer un fonctionnement original en réseau au niveau international, permettant d'allier efficacité et créativité. Les savoir-faire acquis en son sein ont pu être utilisés par les experts dans leur propre pays, faisant progresser les systèmes statistiques et la qualité des instruments d'évaluation. Il est regrettable qu'en France, un nombre encore trop faible de décideurs soient convaincus de l'intérêt de tels échanges entre experts et, plus généralement, de démarches rigoureuses d'évaluation. Désormais, les indicateurs de l'OCDE constituent un thermomètre très prisé, qui joue dans de nombreux pays membres un rôle important dans les choix de politiques éducatives. C'est moins vrai en France. Toutefois, on peut regretter certaines carences dans la stratégie de communication des résultats, notamment vis-à-vis de la presse qui a souvent tendance à exagérer certains résultats et, parfois, à tirer des conclusions péremptoires sur des écarts de résultats statistiquement peu significatifs. Mais ce phénomène existe aussi, il est vrai, au niveau national et local.

Pour un panorama complet de l'évaluation comme instrument de pilotage, il faut au moins mentionner l'évaluation des politiques publiques et des innovations d'une part, l'évaluation des personnels d'autre part. Cette dernière est, dans tous les pays du monde, la moins développée. Il est difficile de préciser, en termes objectifs et généraux, ce qu'est un bon enseignant ou un bon chef d'établissement. La pluralité des contextes fait qu'il y a une pluralité de métiers : être enseignant dans un collège dit «sensible» ne requiert pas nécessairement les mêmes qualités et compétences que pour enseigner dans une classe de terminale d'un lycée prestigieux. Il importe pourtant de trouver une pluralité de critères relativement objectifs, permettant d'apprécier les prestations des personnels, car «l'effet professeur» est loin d'être négligeable. Chacun a pu en faire l'expérience.

En ce qui concerne l'évaluation des politiques publiques, beaucoup reste à faire, même si les méthodologies et les savoir-faire ont progressé. En France, l'exemple de l'évaluation régulière des zones d'éducation prioritaire (ZEP) reste un exemple particulièrement probant en la matière ${ }^{19}$. On peut regretter qu'il n'y ait pas davantage d'évaluations du même type dans d'autres domaines de l'éducation. De même, en matière de réforme ou d'innovation, on peut regretter l'absence d'un principe général d'évaluation systématique des

19 C. Moisan, J. Simon, Les déterminants de la réussite scolaire en ZEP, Paris, INRP, 1997 ; A. Michel, " L'école en quête d'équité ", Administration et éducation, n¹, 1999. 
expérimentations, à une échelle et dans des conditions significatives, avant toute décision de généralisation - même s'il est vrai que ce type de pratique tend à se développer sur la longue période.

\section{Évaluation et gouvernance}

Il apparaît donc une tendance générale à la multiplication des pratiques et des instruments d'évaluation au sein des systèmes éducatifs, ceux-ci étant de plus en plus conçus comme étant au service du pilotage du changement. Nul ne peut nier la plus grande transparence démocratique et les progrès dans la qualité des débats qui en résultent, même si l'on peut craindre un risque excessif de dérive dans l'utilisation d'un discours managérial importé du monde de l'entreprise. En particulier, on peut redouter un instrumentalisme excessif qui porterait à réduire en simples problèmes de gestion des questions politiques fondamentales. De même il existe un risque de nominalisme conduisant à confondre discours rhétorique, modèles, indicateurs statistiques et évolution du réel vécu par les acteurs dans leur quotidien. L'évaluation ne doit être qu'un instrument au service de davantage de démocratie et de concertation, un outil d'aide à la négociation et à la décision. Elle doit donc être utilisée avec précaution et ne pas être sacralisée, au risque de devenir le vecteur de nouveaux comportements technocratiques, ce qui serait particulièrement inacceptable dans ce domaine infiniment humain qu'est l'éducation.

Enfin, l'évolution des démarches d'évaluation est directement liée, dans le domaine de l'éducation comme dans d'autres sphères de l'action publique, aux conflits de pouvoir entre divers acteurs au sein de la recomposition du paysage politique qui accompagne le double mouvement de mondialisation/décentralisation. Celui-ci appelle une nouvelle dialectique local/global qui a conduit à penser la problématique de la régulation et du pilotage en termes de "gouvernance» ${ }^{20}$. L'expertise dans le domaine de l'évaluation et son utilisation pertinente seront de plus en plus au cœur des nouvelles querelles de territoires et des débats sur l'avenir de nos systèmes éducatifs.

$20 \mathrm{Y}$. Dutercq, «Vertus et limites d'un gouvernement local éducatif ", Administration et éducation, $\mathrm{n}^{\circ} 1,1999$; du même auteur, Politiques éducatives et évaluation : querelles de territoires, Paris, Puf, 2000 ; J-L. Derouet, Y. Dutercq, L'établissement scolaire. Autonomie locale et service public, Paris, ESF, 1997. 